\title{
GLAD!
}

Revue sur le langage, le genre, les sexualités

$10 \mid 2021$

Varia

\section{Femmes, artistes et immigrantes : la discrimination multiple à l'égard des écrivaines contemporaines de la diaspora africaine}

Women, Artists and Immigrants: Multiple Discrimination against Contemporary African Diaspora Female Writers

\section{Zoly Rakotoniera Rakotondravelo}

\section{OpenEdition}

Journals

Édition électronique

URL : https://journals.openedition.org/glad/2600

DOI : $10.4000 /$ glad.2600

ISSN : 2551-0819

Éditeur

Association GSL

\section{Référence électronique}

Zoly Rakotoniera Rakotondravelo, «Femmes, artistes et immigrantes : la discrimination multiple à

l'égard des écrivaines contemporaines de la diaspora africaine », GLAD! [En ligne], 10 | 2021, mis en ligne le 01 juillet 2021, consulté le 26 juillet 2021. URL : http://journals.openedition.org/glad/2600 : DOI : https://doi.org/10.4000/glad.2600

Ce document a été généré automatiquement le 26 juillet 2021.

\section{(c) (†) $\odot$}

La revue GLAD! est mise à disposition selon les termes de la Licence Creative Commons Attribution Pas d'Utilisation Commerciale - Pas de Modification 4.0 International. 


\section{Femmes, artistes et immigrantes : la discrimination multiple à l'égard des écrivaines contemporaines de la diaspora africaine}

Women, Artists and Immigrants: Multiple Discrimination against Contemporary

African Diaspora Female Writers

Zoly Rakotoniera Rakotondravelo

1 Depuis le début du XXIe siècle, les femmes auteurs ont dépassé les marges des textes d'écrivains masculins pour occuper une place plus importante dans le canon littéraire africain. L'écriture des femmes africaines a été acclamée par la critique grâce à d'éminents prix, à des romans à succès et à un regard pénétrant sur les expériences des femmes. Un grand nombre de ces écrivaines contemporaines ont immigré dans les pays du Nord, une expérience qui leur confère des possibilités nouvelles et élargies ainsi que des réalités paradoxales de l'altérité.

2 Cet essai explore certains aspects du statut littéraire des écrivaines africaines anglophones de la diaspora en se basant sur la question : comment l'intersection de la race et du genre influence-t-elle l'identité et le statut des écrivaines africaines de la diaspora? Notre point de vue se limite aux romancières et nouvellistes de langue anglaise d'origine africaine qui ont immigré dans les pays du nord.

\section{Les écrivaines dans le canon littéraire africain : des inégalités basées sur le genre}

3 Traditionnellement, le domaine de la fiction africaine a été dominé par des auteurs masculins. Le premier roman publié par une femme africaine, Efuru, de Flora Nwapa est apparu seulement en 1966 au moment où une tradition littéraire masculine distincte était déjà bien établie en Afrique. A cette époque des auteurs masculins tels que Chinua 
Achebe, Ngũgĩ wa Thiong'o et Wole Soyinka ont déjà reçu plus de cinq prix et distinctions internationaux dont le prix Nobel alors que les femmes peinaient à se faire publier.

Un tel écart est dû à des facteurs socio-économiques et culturels sexo-spécifiques, notamment l'accès aux ressources et la répartition des rôles au sein de la famille et de la société. Un auteur doit disposer de capitaux culturels et/ou économiques: être romancière suppose en effet d'avoir fait des études et parfois de maîtriser une langue étrangère.

5 Ensuite les responsabilités domestiques et familiales, dans lesquelles les femmes jouent encore un rôle plus important que celui qu'assument les hommes, sont difficiles à combiner avec les heures de travail longues et irrégulières, et les absences inhérentes à la création, à la production, à la mise en réseau et à la promotion liée à l'écriture.

Le rôle de la critique dans l'invisibilité des femmes dans le canon littéraire au $20^{\mathrm{e}}$ siècle est aussi considérable. Il y a eu un manque d'attention sérieuse voire même une véritable exclusion des écrivaines. Les ouvrages critiques sur les auteures sont très rarement parus, et les études publiées n'incluent pas d'écrivaines ayant publié plusieurs œuvres telles que Bessie Head ou Flora Nwapa. Par exemple la plus grande étude sur la littérature africaine publiée par l'UNESCO en 1987 ne mentionne pas d'écrivaines. (Zulfiqar-Chaudhry $2013: 3$ )

7 Les écrivaines africaines ont lutté pour gagner une plus grande place dans ce canon littéraire et l'immigration a joué un rôle primordial dans cette bataille. En effet, une grande partie de l'écriture africaine contemporaine émane d'écrivains vivant et travaillant en dehors des pays où ils sont nés.

\section{Les enjeux de la littérature de la diaspora féminine}

8 A la fin du $20^{\mathrm{e}}$ et au début du $21^{\mathrm{e}}$ siècle, la migration a engendré un grand changement dans le paysage littéraire africain avec l'émergence d'auteurs tels que Chimamanda Ngozi Adichie, Sefi Atta et No Violet Bulawayo entre autres. Cette nouvelle génération d'écrivaines se caractérise comme «des écrivains post-nationalistes aux préoccupations cosmopolites. Elle est souvent considérée comme ayant le meilleur potentiel "pour se libérer de l'obsession prévisible, presque obligatoire, de l'écrivain africain pour la nation et la politique nationale." » (Habila cité par Iduma 2012 : 4).

9 En effet, pour les écrivaines d'origine africaine, la migration est définie comme une avenue émergente pour transcender les restrictions du nationalisme et représente une position privilégiée rendant possible une agentivité. Souvent associée au concept d'afropolitanisme, cette génération d'auteures dites de la diaspora célèbre une éthique entièrement postcoloniale et post-nationale. Elles sont libres de façonner leur identité et leurs liens avec le continent comme elles l'entendent (culturellement et matériellement), en partie grâce à une liberté de mobilité physique à l'intérieur et entre des multiples sites d'appartenance. (Diop $2016: 16$ )

10 Cependant, l'accès à de nouveaux espaces engendre de nouvelles dynamiques qui parfois ne correspondent pas aux idéaux mentionnés auparavant. Qu'en est-il alors des formes d'altérité dont ces écrivaines font l'expérience?

11 Dans son écrit sur le statut de l'écrivain de la diaspora, le romancier britannique Salman Rushdie dit que ce dernier est généralement "considéré avec suspicion ». 
(Philippe 2014 : 1). Une telle suspicion s'applique communément à l'ensemble des écrivains migrants, et devient ainsi la source d'une classification spécifique dans la littérature mondiale. En effet, les écrivains migrants, et plus précisément, les écrivains originaires d'anciens pays colonisés, font l'objet de catégorisations parfois abusives comme "ghettoïsation", et d'essentialisation. (Mathis-Moser \& Mertz-Baumgartner $2014: 50)$.

Dans son article "arrêter de cataloguer les écrivains africains ", la romancière Tayie Selasi (2015) demande «Pourquoi les écrivains africains doivent-ils toujours porter le fardeau de représenter leur continent? La liberté artistique devrait leur être accordée, comme elle l'est aux autres auteurs ». Elle analyse plusieurs points sur la ghettoïsation des auteurs qui est en partie due aux médias et au système en général. Elle attribue cette situation à l'attachement des médias à ce qu'elle nomme sarcastiquement "des petites tendances ethniques. " Elle pose alors la question sur ce que les romans dits africains ont en commun : la catégorie commerciale fonctionne-t-elle aussi comme une catégorie créative? (Selasi 2015)

Une autre romancière, Aminatta Forna, dans une interview récente demande pourquoi son roman The Hired Man (2013) qui se déroule en Croatie, se trouve toujours dans la «section africaine » des librairies. Forna cite d'autres romans tels que Boy, Snow, Bird (2014) d'Helen Oyeyemi, qui se passe en Nouvelle-Angleterre, ou l'Open City (2011) de Teju Cole, dont une grande partie a lieu à Manhattan et se demande si ce sont des œuvres africaines?

Mais c'est l'autrice Yewande Omotoso, qui le plus clairement dénonce l'incapacité générale de voir au-delà des catégories. Dans son manifeste intitulé «L'épidémie de penser qu'il n'y a qu'un seul écrivain africain" (Literary Hub 2019), elle raconte comment elle a fait l'expérience de ce cloisonnement. Lors d'une rencontre littéraire en Europe, l'auteure a dû parler de la façon dont l'Afrique a influencé son écriture. Sa réaction était de se demander pourquoi devions-nous expliquer notre travail exclusivement sur la base d'un aspect de notre identité. Elle conclut son manifeste sur l'affirmation que la manière dont nous rangeons les gens dans des compartiments montre que nous ne savons pas comment voir, nous voyons ce qui convient à notre image et éludons ce qui ne correspond pas à cette image.

Ces questionnements relatifs à l'impact de l'immigration sur l'identité des auteurs de la diaspora africaine sont renforcés par Chimamanda Ngozi Adichie. La romancière affirme que pour elle,

voyager avec un passeport nigérian, c'est porter le poids des hypothèses. Cela signifie être, à de nombreux points d'entrée, automatiquement suspect. Voyager avec un passeport nigérian, c'est constamment faire face à l'incrédulité moqueuse des agents d'immigration quand je dis que je suis écrivaine, c'est se faire demander de se retirer pour plus de questions, c'est se sentir coupable de quelque chose. (New Statesman 2019)

\section{Les stratégies d'intervention}

Pour un monde littéraire plus égalitaire, il est nécessaire de lutter pour mettre un terme à ces formes de discriminations basées sur la race et le genre. Une des actions qui ont largement donné de l'élan à la littérature africaine depuis le début du $21^{\mathrm{e}}$ siècle est la création du prix Caine pour l'écriture africaine. C'est un prix littéraire décerné 
depuis 2000 chaque année pour la meilleure nouvelle en langue anglaise publiée en Afrique ou ailleurs par un écrivain africain. Les objectifs du Prix ainsi que les principes sur lesquels il est attribué peuvent être considérés comme des actions visant à éliminer les inégalités mentionnées auparavant.

Premièrement, le Prix Caine permet à une nouvelle génération d'écrivains d'émerger, notamment en leur donnant accès à une industrie assez fermée et, ce qui est important, en accordant une somme d'argent décente avec laquelle gagner du temps pour écrire. L'attribution du prix tient particulièrement compte du genre et de l'égalité des chances.

Deuxièmement, le prix Caine a pour objectif d'encourager la reconnaissance de la valeur de l'écriture africaine en anglais, de sa richesse et de sa diversité, en l'apportant à un public plus large. (Attree 2013 : 36). En effet une grande partie des autrices ont acquis une certaine notoriété soit en remportant le prix, soit en étant présélectionnées pour le prix.

19 Un autre critère de sélection du prix Caine qui est très important est le fait qu'il ne tient pas compte du sujet de l'œuvre, n'exige pas que le sujet ait une relation avec l'Afrique, c'est la capacité de l'auteur à faire une interprétation "réussie d'un événement ou d'un moment particulier dans le temps en utilisant le langage " qui obtient l'approbation des juges. (Attree $2013: 38$ ).

Ce genre d'effort pourrait permettre aux femmes écrivains émergentes qu'elles soient immigrantes ou non de percer dans l'espace littéraire et d'éliminer les inégalités liées aux genres et à la race dont elles peuvent être victimes. Une autre action un peu plus simple et qui est à la portée de tout un chacun est de continuer à dénoncer la ghettoïsation des auteurs immigrants de façon systématique comme le font les auteurs mentionnés dans cet essai. En effet, l'auteure Amy Tan, un mois après avoir publié son article qui dénonce le cloisonnement des auteures asiatico-américains a été incluse dans les anthologies de la littérature américaine. (Schultermandl $2008: 290$ )

Pour conclure, être femme et immigrante se traduit par plusieurs formes de discrimination et d'inégalité pour les auteures de la diaspora anglophone africaine. Pourtant, c'est la diversité et la richesse des identités des auteures qui a de plus en plus alimenté la fiction africaine contemporaine. C'est une situation qui nécessite une action plus concertée et plus systématique.

\section{BIBLIOGRAPHIE}

ADICHIE, Chimamanda Ngozi. 2019. «Shut up and write ». New Statesman. https:// www.newstatesman.com/culture/books/chimamanda-ngozi-adichie-african-writer-artcitizenship-essay

ATTREE, Lizzy. 2013. " The Caine Prize and Contemporary African Writing ». Research in African Literatures, 44(2). DOI:10.2979/reseafrilite.44.2.35. 
DIOP, Papa Samba. 2016. « Des mots et concepts nouveaux en circulation dans l'espace francophone : l'“Afropolitanisme” en question ». Nouvelles Études Francophones 31(2). DOI:10.1353/ nef.2016.0036.

IDUMA, Emmanuel. 2013. « Notes on Writing from Within ». The Mantle, consulté le 16 Août 2020. URL : https://www.themantle.com/arts-and-culture/notes-writing-within

MATHIS-MOSER, Ursula. \& MERTZ-BAUMGARTNER, Birgit. 2014. « Littérature migrante ou littérature de la migrance : À propos d'une terminologie controversée ». Diogène, 246-247(2). DOI: 10.3917/dio.246.0046.

OMOTOSO, Yewande. 2019. « The Epidemic of Thinking There's Only One African Writer. » Literary Hub consulté le 13 juillet 2020. URL : https://lithub.com/what-to-do-when-youre-mistaken-foranother-writer/

PHILIPPE, Nathalie.2014. « Écrivains migrants, littératures d'immigration, écritures diasporiques ». Hommes \& migrations. URL : http://journals.openedition.org/hommesmigrations/ 1543

SCHULTERMANDL, Silvia. 2008. (Breaking out of) the "Literary Ghetto": Where to Place Asian American Writers, Or De-essentializing Canon Formation. Hungarian Journal of English and American Studies (HJEAS), 14(2). DOI:www.jstor.org/stable/41274431

SELASI, Tayie. 2015. « Stop pigeonholing African writers ». The Guardian consulté 25 juillet 2020. URL : https://www.theguardian.com/books/2015/jul/04/taiye-selasi-stop-pigeonholing-africanwriters

ZULFIQAR CHAUDHRY, Sadia. 2014. African women writers and the politics of gender. Thèse de Doctorat. http://theses.gla.ac.uk/5202/

\section{RÉSUMÉS}

Les écrivaines africaines ont lutté pour gagner une plus grande place dans le canon littéraire et l'immigration a joué un rôle primordial dans cette bataille. En effet, une grande partie de l'écriture africaine contemporaine émane d'écrivains vivant et travaillant en dehors des pays où ils sont nés. Cet essai explore certains aspects du statut littéraire des écrivaines africaines anglophones de la diaspora en se basant sur la question : comment l'intersection de la race et du genre influence-t-elle l'identité et le statut des écrivaines africaines qui ont immigré dans les pays du Nord? En effet, l'accès à de nouveaux espaces engendre de nouvelles dynamiques qui leur confèrent des possibilités nouvelles et élargies ainsi que des réalités paradoxales de l'altérité. C'est une situation qui nécessite une action plus concertée et plus systématique. Une des stratégies qui peuvent servir de modèle pour lutter contre les discriminations est le Prix Caine. Les objectifs du Prix ainsi que les principes sur lesquels il est attribué peuvent être considérés comme des actions visant à éliminer les inégalités de race et de genre dont les autrices de la diaspora africaine peuvent être victimes.

African women writers have struggled to gain a greater place in the literary canon, and immigration has played a key role in this battle. Indeed, much of contemporary African writing emanates from writers living and working outside the countries in which they were born. This essay explores aspects of the literary status of English-speaking African women writers in the diaspora based on the question: How does the intersection of race and gender influence the identity and status of African women writers who have immigrated to Northern countries? Indeed, access to new spaces generates new dynamics that provide them with new and expanded 
opportunities and the paradoxical realities of otherness. It is a situation that requires more concerted and systematic action. One of the strategies that can serve as a model to fight discrimination is the Caine Prize. The objectives of the Prize as well as the principles on which it is awarded can be seen as actions aimed at eliminating racial and gender inequalities of which the female members of the African Diaspora may be victims.

\section{INDEX}

Keywords : inequality, gender, race, intersectionality, immigration, literature

Mots-clés : inégalités, genre, race, intersectionnalité, immigration, littérature

Thèmes : Explorations

\section{AUTEUR}

\section{ZOLY RAKOTONIERA RAKOTONDRAVELO}

Zoly Rakotoniera Rakotondravelo est maîtresse de conférences au Département d'études anglophones et des études de genre à la Faculté des Lettres et Sciences Humaines de l'Université d'Antananarivo. Elle a publié de nombreux travaux sur la condition féminine, le genre, les cultures et les sociétés du Sud et du Nord. Elle participe à plusieurs projets de recherche notamment à l'élaboration d'un ouvrage collectif sur la représentation de la figure de la mariée dans la littérature et les médias. 